\title{
Implementation of an LES mixed subgrid model for the numerical investigation of flow around a circular cylinder at $R e=3,900$ and 140,000
}

\author{
J. Wong \& E. Png \\ Marine Systems, DSO National Laboratories, Singapore
}

\begin{abstract}
Flows around ships, autonomous underwater vehicles, and other marine structures are often complicated and highly three-dimensional. For accurate flow prediction, large eddy simulation (LES) is a promising alternative which is more accurate than Reynolds-averaged (RANS) turbulence modelling and computationally less intensive than direct numerical simulation (DNS). The flow over a cylinder at $\mathrm{Re}=3900$ and $\mathrm{Re}=140,000$ is investigated using the commercial Ansys-Fluent CFD solver and the performance of two different LES subgrid models is evaluated. In particular, we have incorporated the scale similarity term of Bardina into the conventional Smagorinsky model using UserDefined Functions, to form what is known as a Mixed subgrid model. The Mixed subgrid model gives the best agreement with measurements, especially for higher Reynolds numbers where the grid is correspondingly coarser with respect to the flow. This has important implications for the LES modelling of marine engineering applications, where the Reynolds numbers involved are typically extremely large and fine meshes are computationally expensive.
\end{abstract}

Keywords: large eddy simulation, cylinder, mixed subgrid model, scale similarity, smagorinsky, FLUENT, user-defined functions.

\section{Introduction}

Flows around ships, autonomous underwater vehicles, and other marine structures are often complicated and highly three-dimensional. The traditional approaches of using facility testing to measure the performance of marine vessels 
have been complemented with computational fluid dynamics predictions in recent years. Simulations have the benefits of cost-savings and also serve to elucidate on certain details of flow physics which cannot be easily measured. For highly unsteady turbulent flows, large anisotropic eddies are generated which are important in the turbulent transport of flow variables. A wide range of time and length scales are involved and Reynolds-averaged (RANS) models are often unable to give accurate predictions in such situations. These situations might include instances when the marine vessel is undergoing turning maneuvers, or when the flow noise, both self- and radiated, needs to be computed. Theoretically, direct numerical simulation is able to resolve all the length and time scales inherent in the flow; however, the computational cost for practical simulations would be exorbitant. Large Eddy Simulation (LES) is a promising approach in which the large eddies are accurately resolved while the smaller eddies are modeled via a subgrid turbulent model. Thus it is able to predict the flow more accurately than RANS methods while providing cost savings compared to DNS.

The flow around a circular cylinder at various Reynolds numbers has been the subject of extensive measurements and numerical studies. Although the geometry is simple, it possesses flow features which are common in many marine structures such as submarine periscopes, ship rudders, marine pipelines, and offshore platform support legs. Such flows typically include curved boundary layers, boundary layer separation, laminar-turbulent transition, unsteady shear layers, and vortex shedding. It is therefore important to be able to predict the flow around the circular cylinder at high Reynolds numbers accurately. To this end, numerous experimental studies have been conducted [1-11].

With the advent in computing power over the years, the use of LES for engineering applications is becoming increasingly common [12-15]. The practicing engineer in industry can now turn to commercially available CFD software such as Ansys-Fluent, for their LES computing needs [16]. These solvers contain robust numerics, a wide range of prediction modules, as well as user-friendly graphical user-interfaces for easy usage. However, the LES models available on the commercial market currently are solely of the pure eddyviscosity type. These functional models (see section 2.1) are unable to adequately capture the flow physics, especially at the high Reynolds numbers which are of practical importance to industry. To this end, we have incorporated a structural model, known as a Mixed subgrid model, which contains the scalesimilarity term of Bardina's. This, and the constant-coefficient Smagorinsky model, which is available in FLUENT, are then tested for the flow past a circular cylinder at two Reynolds numbers, $\mathrm{Re}=3900$ and $\mathrm{Re}=140,000$.

\section{LES modelling technique}

In LES the flow is divided into two regimes $\mathbf{v}=\overline{\mathbf{v}}+\mathbf{v}^{\prime}$, where $\overline{\mathbf{v}}(\mathbf{x}, t)=G * \mathbf{v}(\mathbf{x}, t)$ denotes the large-scale (resolvable) component and $\mathbf{v}^{\prime}(\mathbf{x}, t)$ 
the small-scale component. Here, $G=G(\mathbf{x}, \Delta)$ is a filter function and $\Delta(\mathbf{x})$ is the characteristic grid spacing. Convolving the incompressible NSE with G yields,

$$
\partial_{t}(\overline{\mathbf{v}})+\overline{\nabla \cdot(\mathbf{v} \otimes \mathbf{v})}=-\overline{\nabla p}+\overline{\nabla \cdot \mathbf{S}}+\overline{\mathbf{f}}, \quad \overline{\nabla \cdot \mathbf{v}}=0
$$

where $\mathbf{v}$ is the velocity, $\mathrm{p}$ the pressure, $\mathbf{S}=2 v \mathbf{D}$ the viscous stress tensor, $v$ the viscosity, $\mathbf{D}=\frac{1}{2}\left(\nabla \mathbf{v}+\nabla \mathbf{v}^{T}\right)$ the rate-of-strain tensor and $\mathbf{f}$ the body force. Recognizing that filtering and derivative operators do not generally commute, i.e. $[G *, \nabla] \rho=\overline{\nabla \rho}-\nabla \bar{\rho} \neq 0,[17]$, and that the advection term in (1) gives rise to unclosed terms,

$$
\partial_{t}(\overline{\mathbf{v}})+\nabla \cdot(\overline{\mathbf{v}} \otimes \overline{\mathbf{v}})=-\nabla \bar{p}+\nabla \cdot(\overline{\mathbf{S}}-\mathbf{B})+\overline{\mathbf{f}}+\mathbf{m}, \quad \nabla \cdot \overline{\mathbf{v}}=m,
$$

where $\quad \mathbf{B}=(\overline{\mathbf{v} \otimes \mathbf{v}}-\overline{\mathbf{v}} \otimes \overline{\mathbf{v}}) \quad$ is the subgrid stress tensor and $\mathbf{m}=[G *, \nabla](\mathbf{v} \otimes \mathbf{v}+p \mathbf{I}-\mathbf{S})$ and $m=[G *, \nabla] \mathbf{v}$ are the commutation error terms. Although both terms contribute to the closure problem of LES, these are often neglected since they are expected to be smaller than the effect of the unresolved transport.

\subsection{Subgrid stress modelling}

Many analyses performed in the framework of fully isotropic turbulence reveal two main mechanisms which govern the interactions between large and small scales: the forward energy cascade, in which the subgrid scales drain energy from the resolved scales, and the backward energy cascade, in which there is a weak feedback of energy, proportional to $k^{4}$, to the resolved scales. Correspondingly, there exist two main modelling approaches. In functional modelling, it is assumed that the action of the subgrid scales on the resolved scales is mainly energetic in nature so that the balance of the energy transfers between the two scale ranges is sufficient to describe the subgrid scale effects. Structural modelling focuses on modelling B without incorporating any knowledge of the nature of interactions between subgrid and resolved scales [18]. Most LES modelling for high Reynolds flows today are of the functional type and are able to account for the forward energy cascade while structural models (based on either scale similarity or mixed subgrid models, series expansion techniques, and other techniques) which attempt to model the backscatter of energy from the subgrid to the resolved scales, are not widely used [17]. Although structural models are able to capture more of the relevant flow physics, they are not popular because the same mechanism which predicts the backward cascade can sometimes lead to numerical instability. Here we will compare the performance of two LES models, the conventional Smagorinsky model belonging to the class of functional models, and the Mixed subgrid model 
belonging to the class of structural models, incorporated via the use of FLUENT User-Defined Functions.

\subsubsection{Smagorinsky model}

The Smagorinsky model is an eddy-viscosity type model which models the subgrid stress tensor B using the Boussinesq hypothesis:

$$
\mathbf{B}=-2 v_{s g s} \overline{\mathbf{D}}
$$

where $v_{s g s}$ is the subgrid viscosity and $\overline{\mathbf{D}}$ is the resolved rate-of-strain tensor. The subgrid viscosity is in turn calculated as

$$
v_{s g s}=\left[\min \left(0.42 d, C_{s} V^{1 / 3}\right)\right]^{2}\left|C_{s} \sqrt{2 \overline{D_{i j} D_{i j}}}\right|
$$

where $\mathrm{d}$ is the distance from the closest wall, $\mathrm{V}$ is the volume of the computational cell, $\overline{D_{i j}}$ is the rate of strain tensor, and $C_{s}$ is the Smagorinsky constant with a default value of 0.1 [19].

\subsubsection{Mixed subgrid model}

The use of the Boussinesq hypothesis in the Smagorinksy subgrid model means that $\mathbf{B}$ is necessarily aligned with $\overline{\mathbf{D}}$. However, tests performed on databases generated by DNS have shown that there is in fact a low level of correlation between the two tensors. Bardina's model, in which the subgrid tensor is calculated by applying the filter twice, shows a much better correlation [17]. In our implementation, following Fureby et al. [20], we first define the subgrid velocity as $\mathbf{v}^{\prime}=\mathbf{v}-\overline{\mathbf{v}}$. Applying the filter a second time gives $\overline{\mathbf{v}^{\prime}}=\overline{\mathbf{v}}-\overline{\mathbf{v}}$. Then B can be further decomposed as

$$
\begin{aligned}
& \mathbf{B}=(\overline{\overline{\mathbf{v}} \otimes \overline{\mathbf{v}}}-\overline{\overline{\mathbf{v}} \otimes \overline{\mathbf{v}}})+\left(\overline{\overline{\mathbf{v}} \otimes \mathbf{v}^{\prime}}-\overline{\overline{\mathbf{v}}} \otimes \overline{\mathbf{v}^{\prime}}+\overline{\mathbf{v}^{\prime} \otimes \overline{\mathbf{v}}}-\overline{\mathbf{v}^{\prime}} \otimes \overline{\mathbf{v}}\right)+\left(\overline{\left(\mathbf{v}^{\prime} \otimes \mathbf{v}^{\prime}\right.}-\overline{\mathbf{v}^{\prime}} \otimes \overline{\mathbf{v}^{\prime}}\right) \\
& =\mathbf{L}+\mathbf{C}+\mathbf{R}
\end{aligned}
$$

where $\mathbf{L}$ is the modified Leonard term, and $\mathbf{C}+\mathbf{R}$ is modelled by a subgrid viscosity model; for this, we choose the Smagorinsky model (Eqns (3) and (4)). For practical reasons, it is also necessary to include this because tests have shown that the modified Leonard term alone is only slightly dissipative, and therefore might not be able to drain sufficient energy from the resolved scales, resulting in an underestimation of the forward energy cascade and numerical instability. The Leonard term appears as an explicit source term in the LES momentum equations, and linear interpolation is used to obtain the twice filtered velocities.

\subsection{Near-wall treatment}

Special treatment is needed to address the boundary layer flow due to the formation and growth of small but important eddies in the near-wall region. Two 
approaches exist: the first consists of resolving the near-wall flow directly. In practice, this requires the first computational cell to be within the viscous sublayer. However, this is clearly not possible for high Reynolds number flows, and thus we have to rely on modelling the wall dynamics instead. A number of such wall-models exist: these include Deardorff's model, which work by enforcing boundary conditions on second-order derivatives, and wall stress models, which work by modifying the subgrid viscosity of the LES models. Several such models exist, for example the Schumann model, used with good results for the case of an inclined prolate spheroid by Wikstrom et al. [21], the Grotzbach model, the model of Werner and Wengle, which is available in FLUENT, and others. A third class of wall-models modify the subgrid viscosity according to the distance from the wall to provide the correct scaling; a well known example is the Van Driest's damping function.

As the purpose of the simulations is to highlight the effect of the scalesimilarity term, we use FLUENT's ad-hoc wall model (belonging to the third class of wall models) which comes with the basic Smagorinsky model. In this wall model, the turbulent viscosity is reduced for the first cell adjacent to the wall in the manner

$$
v_{s g s}=\left[\min \left(0.42 d, C_{s} V^{1 / 3}\right)\right]^{2}\left|C_{s} \sqrt{2 \overline{D_{i j} D_{i j}}}\right|
$$

through the wall-distance variable $d$.

\subsection{Numerical methods}

FLUENT uses a finite volume method and a segregated approach to discretize and solve the incompressible LES equations. Pressure-velocity coupling is achieved using the PISO scheme, and the equations are iterated using a secondorder spatial accurate central-differencing scheme and a second-order accurate implicit time-stepping scheme [refer to 19 for details]. It is known that centraldifferencing schemes give rise to numerical oscillations on coarse grids; however, for the current simulations, these oscillations do not seem to affect the computed results in any significant manner. The residuals drop to at least $10^{-6}$ at the end of each time step, and the Courant number, defined here as $C o=V \Delta t / \Delta x$, is kept below 0.8 to ensure a good resolution of the temporal flow dynamics.

\section{Computational set-up}

The computational domain used is rectangular, with the inflow set at $10 \mathrm{D}$ (cylinder diameters) upstream of the cylinder and the outlet at $20 \mathrm{D}$ downstream (see fig. 1). The vertical extent is $20 \mathrm{D}$, giving a blockage ratio of $5 \%$. The spanwise extent of the domain is $\pi \mathrm{D}$. This has the same dimensions as that used by Franke and Frank [22], and Persson et al. [23], amongst others. The velocity is set to a fixed value at the inflow, while a zero gradient is enforced at the outlet. The top and bottom of the domain are slip walls, while the spanwise planes are set as symmetry planes. Finally, the cylinder itself is set to a no-slip wall 


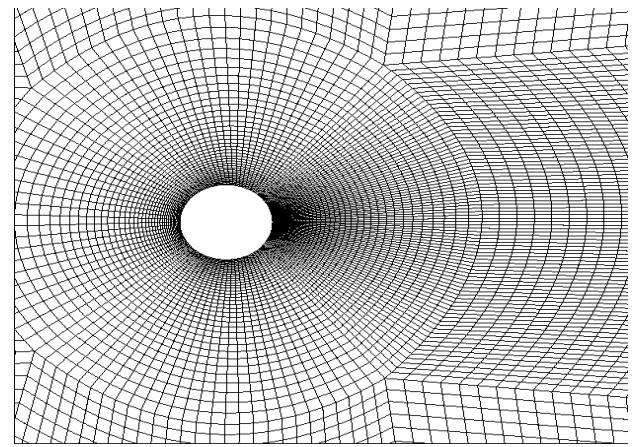

Figure 1: Close-up view of computational mesh close to cylinder.

boundary condition. The cells are clustered in the vicinity of the cylinder as well as the wake, and the grid is stretched in the direction normal to the cylinder wall with a stretching factor of 1.08. The total number of cells used in the domain is 1.1 Million, which gives a near-wall resolution of $\mathrm{y}^{+} \sim 8$ for $\operatorname{Re}=3900$ and $\mathrm{y}^{+} \sim 100$ for $\mathrm{Re}=140,000 . \mathrm{A} \mathrm{y}^{+}$of 100 is chosen (instead of a finer grid) for the higher Re simulations because this is typical (and necessary) for practical LES computations in which compromises have to be made between grid sizes and computational time. It is also thought that the coarse grid would provide a better comparison of the different subgrid models used.

\section{Results and discussion}

\subsection{General flow physics}

Figure 2. shows a perspective view of the iso-surfaces of the second invariant of the velocity gradient $\mathrm{Q}$ for the $\mathrm{Re}=3900$ flow case. It can be seen that the wake separating from the cylinder consists of curved shear layers with vortices being shed from alternate sides of the cylinder. The initial vortical structures consist of spanwise rollers resulting from the Kelvin-Helmholtz instability in the shear layers. Further downstream, these undergo helical pairing and vortex stretching to form longitudinal vortices which persist as far as 10D behind the cylinder. The $\mathrm{Re}=140,000$ flow is similar, although the wake is now smaller due to later flow separation. Figure 3 shows the time-averaged streamlines on the centre plane for $\operatorname{Re}=3900$ and $\operatorname{Re}=140,000$. It is seen that at the lower Reynolds number, there is not much difference between the models. At the higher Reynolds number, it is seen that the constant-coefficient Smagorinsky model overpredicts the extent of the recirculation region, while the Mixed subgrid model gives better agreement with experiment. This is related to the wake statistics and will be further discussed in the following section. 


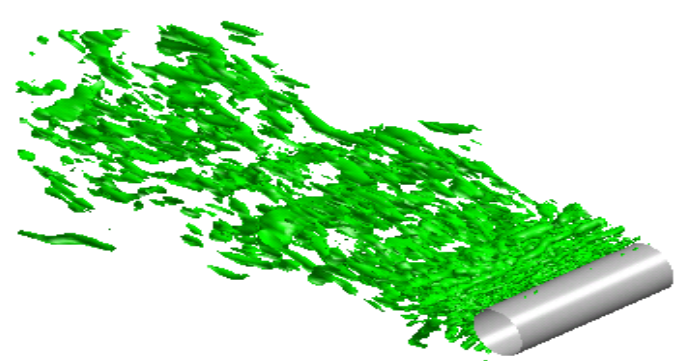

Figure 2: $\quad$ Iso-surfaces of Q behind cylinder for $\mathrm{Re}=3900$.

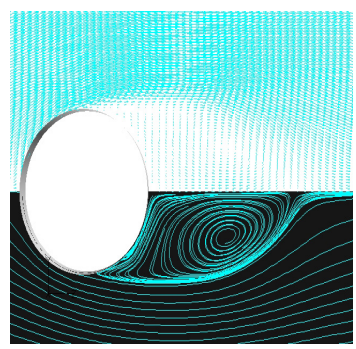

(a)

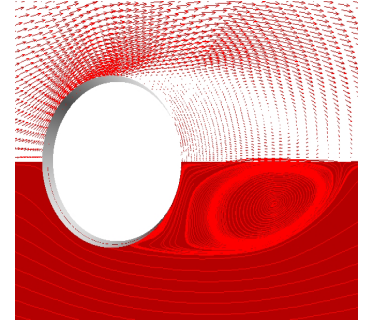

(b)

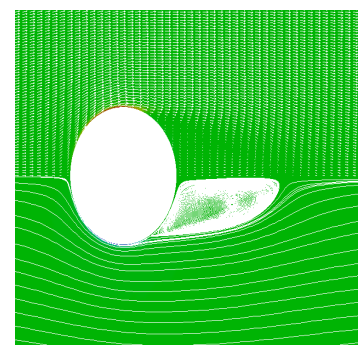

(c)

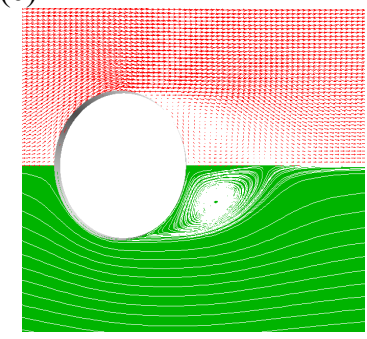

(d)

Figure 3: Time-averaged streamlines on centre plane for $\mathrm{Re}=3900$ and $\mathrm{Re}=$ 140,000 for smagorinsky (a, c) and mixed subgrid models (b, d).

\section{Wake statistics}

4.2

Time averaged profiles of the streamwise velocity are plotted in fig. 4 . For Re $=$ 3900, comparisons are made at locations given by Lourenco and Shih [6] and Ong and Wallace [8]. At the first measurement position, $\mathrm{x} / \mathrm{D}=1.06$, the velocity profile is U-shaped, which is an indication that a sufficiently long sampling time for the velocity has been achieved. Good agreement is obtained in terms of both the centreline velocities and the width of the wake at the first two downstream positions, $\mathrm{x} / \mathrm{D}=1.06$ and $\mathrm{x} / \mathrm{D}=1.54$, whereas LES overpredicts the centreline velocity at about $3 \%$ for the position $\mathrm{x} / \mathrm{D}=2.02$. Further downstream, good agreement is again obtained between LES and experiment. That the width of the 


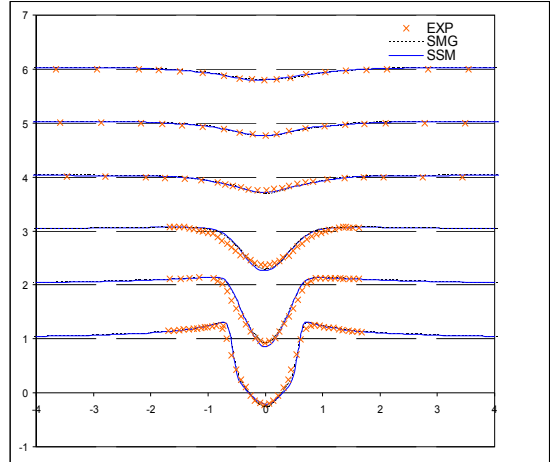

(a)

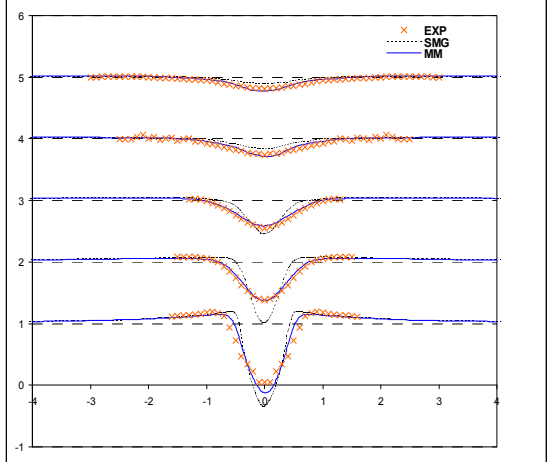

(b)

Figure 4: Time-averaged streamwise velocity at $\mathrm{x} / \mathrm{D}=1.06,1.54,2.02,4,7$ and 10 for (a) $\operatorname{Re}=3900$ and $\mathrm{x} / \mathrm{D}=1,1.5,2,4$ and 7 for (b) $\mathrm{Re}=$ 140,000 .

predicted wake is in good agreement with experiment is also an indication that the flow separation angle on the cylinder is captured correctly. The difference between the conventional Smagorinsky model and the Mixed subgrid model is negligible, indicating that the effect of the subgrid model is not so important at this low Reynolds number. For $\mathrm{Re}=140,000$, comparisons are made with the measurements by Cantwell and Coles [5]. Significant differences between the subgrid models can now be seen. At stations $\mathrm{x} / \mathrm{D}=1,1.5$, and 2, the conventional Smagorinsky model greatly underpredicts the magnitude of the centreline velocity and also predicts a wake which is much too narrow compared to the experiment. This under-prediction of the centreline velocity results in a computed recirculation region that is much larger than measured, as noted in the previous section. The Mixed subgrid model shows better agreement with experimental data, although it still slightly underpredicts the centreline velocity at $\mathrm{x} / \mathrm{D}=1$. The wake width is also captured more accurately, which indicates that the predicted flow separation angle is also closer to experiment. Here, the computational grid is the same one that was used for $\mathrm{Re}=3900$. As a result, the grid is correspondingly coarser with respect to the much higher Reynolds number. The influence of the subgrid model is thus more important.

The streamwise rms velocity fluctuations are presented in fig. 5 . For $\mathrm{Re}=$ 3900, we again see negligible differences between the LES models and experiment. In addition, the measured profiles are slightly asymmetrical, which perhaps indicates that the experimental statistics is insufficient. At $R e=140,000$, a significant difference between the LES models can be seen. The Mixed subgrid model overpredicts the peak rms values while the conventional Smagorinsky model underpredicts it. However, the width of the rms fluctuations is much better captured by the Mixed subgrid model, while the conventional Smagorinsky model consistently gives a too-narrow wake. In addition, the computed LES profiles show a bimodal character which is also seen for 


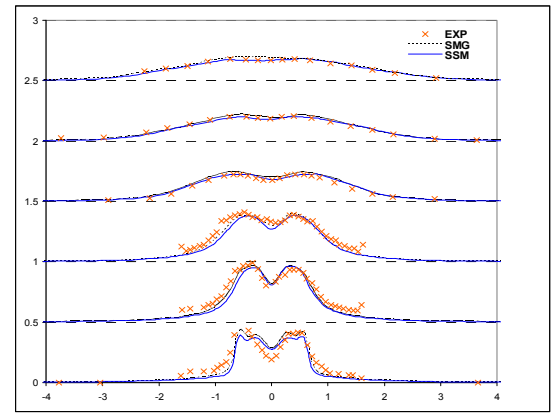

(a)

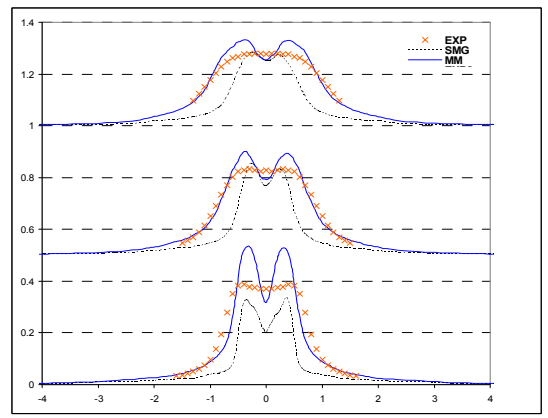

(b)

Figure 5: Time-averaged streamwise rms fluctations for $\mathrm{x} / \mathrm{D}=1.06,1.54$, 2.02, 4, 7 and 10 for (a) $\mathrm{Re}=3900$ and $\mathrm{x} / \mathrm{D}=1,1.5$ and 2 for (b) $\operatorname{Re}=140,000$.

$\operatorname{Re}=3900$, whereas this is absent in the measured profiles. One would expect this bimodal character as it represents velocity fluctuations in the shear layer.

\subsection{Mean integrated quantities}

Next, the mean integrated quantities are shown in table 1. The following quantities are compared:

$$
\begin{gathered}
\mathrm{CP}=\frac{\iint C_{p} \cos \theta d \theta d z}{L}, \\
-\mathrm{C}_{\mathrm{pb}}=\frac{p_{\theta=\pi}-p_{r e f}}{\frac{1}{2} \rho U_{r e f}^{2}} .
\end{gathered}
$$

$\mathrm{L}$ is the length of the cylinder, taken to be $\pi \mathrm{D}$ in this case. For the calculation of the skin friction coefficient, it is assumed that the first cell adjacent to the wall is embedded well within the viscous sublayer since the wall $\mathrm{y}^{+} \sim 8$ for $\mathrm{Re}=3900$. Employing the law of the wall, we have :

$$
\left\langle\tau_{w}\right\rangle=\frac{\mu\langle\bar{U}\rangle}{y} .
$$

At $\operatorname{Re}=140,000$, the wall $\mathrm{y}^{+}$is $\sim 100$, i.e., the log-law region. Hence no similar simple formula for obtaining the mean shear stress is available during postprocessing. It is seen that for $\mathrm{Re}=3900$, both LES models overpredict slightly the mean integrated pressure and the base pressure coefficient, with the Mixed subgrid model giving results that are slightly closer to experiment. Both 
Table 1: $\quad$ Mean integrated cylinder quantities.

\begin{tabular}{|c|c|c|c|c|}
\hline $\mathrm{Re}=3900$ & $\mathrm{CP}$ & $-\mathrm{C}_{\mathrm{pb}}$ & $\phi_{\mathrm{s}}\left(^{0}\right)$ & $\mathrm{L}_{\mathrm{r}} / \mathrm{D}$ \\
\hline Smagorinsky & 1.047 & 1.05 & 94.5 & 1.1332 \\
\hline $\begin{array}{c}\text { Mixed subgrid } \\
\text { model }\end{array}$ & 0.987 & 0.99 & 97.4 & 1.2085 \\
\hline $\mathrm{Expt}$ & 0.942 & 0.84 & 85 & 1.1583 \\
\hline $\mathrm{Re}=140,000$ & $\mathrm{CP}$ & $-\mathrm{Cpb}$ & $\phi \mathrm{s}\left({ }^{0}\right)$ & $\mathrm{Lr} / \mathrm{D}$ \\
\hline Smagorinsky & 0.4673 & 0.589 & - & 0.997 \\
\hline $\begin{array}{c}\text { Mixed subgrid } \\
\text { model }\end{array}$ & 0.9491 & 1.15 & - & 0.620 \\
\hline Expt & 1.2346 & 1.21 & 78 & 0.4421 \\
\hline
\end{tabular}

models overpredict the mean flow separation angle, but not to the extent where the computed wakes differ significantly from the experimental results. The computed recirculation lengths are also in good agreement with experiment, with the conventional Smagorinsky model slightly underpredicting, and the Mixed subgrid model slightly overpredicting, the actual value. At $\operatorname{Re}=140,000$, significant differences can be seen between the two LES models. The integrated pressure coefficient predicted by the Smagorinsky model is more than $50 \%$ lower than the actual value, while the Mixed subgrid model gives a much better result with around $20 \%$ discrepancy. The differences become even more significant when one compares the base pressure coefficients. Again, the Smagorinsky model computes a value which differs from the measured by more than 50\%, while the Mixed subgrid model gives much better agreement, predicting a value which is only 5\% lower than the experimental result. Likewise, the recirculation length predicted by the Smagorinsky model exceeds the measured value significantly, in part due to the high value of Cs $(=0.1)$ used in the current computations, as noted by Ma et al. [7]. The Mixed subgrid model is able to rectify this to a certain degree, and reduces the computed recirculation length discrepancy from $\mathrm{L}_{\mathrm{r}} / \mathrm{D}=0.997$ to $\mathrm{L}_{\mathrm{r}} / \mathrm{D}=0.620$, which is more in line with the measured value of $\mathrm{L}_{\mathrm{r}} / \mathrm{D}=0.4421$.

\section{Conclusions}

In this study, we have performed Large Eddy Simulations of the flow over a cylinder for $\mathrm{Re}=3900$ and $\mathrm{Re}=140,000$. We compare the predictive capability of the constant-coefficient Smagorinsky model with the Mixed subgrid model, which consists of an additional Leonard stress tensor term. For the lower Reynolds number, the agreement between LES models and experiment is very good. This implies that most of the energetic vortices are fully resolved by the grid and therefore the difference between subgrid models is small. At the higher Reynolds number of 140,000, significant differences exist between LES models and experiment. In particular, the proposed Mixed subgrid model gives flow prediction which are in much better agreement with experimental data. The predicted time-averaged streamwise velocity is in good quantitative agreement and while the rms fluctuations overpredict the peak values, the mixed subgrid model is at least able to capture the width of the wake. The recirculation length 
and the mean integrated cylinder quantities are also much better predicted. For example, the integrated pressure coefficient predicted by the mixed subgrid model has a discrepancy of $20 \%$ while the Smagorinsky model differs by more than $50 \%$, and the base pressure coefficient computed by the mixed subgrid model differs from experiment by only $5 \%$ while the Smagorinsky value differs by more than $50 \%$. The reason for this improvement is thought to be due to the handling of the smallest resolved scales, as well as the inter-scale energy transfer, in particular the backward energy cascade, offered by the Leonard stress tensor term. This has important practical implications for the ability to conduct high Reynolds number LES computations. This mixed subgrid model will allow the practicing engineer to conduct good quality hydrodynamic analysis at a fraction of the computational cost that would be required for a much finer mesh if the constant-coefficient Smagorinsky model is used.

\section{References}

[1] Achenbach E., Distribution of local pressure and skin friction around a circular cylinder in cross-flow up to $\mathrm{Re}=5 \times 10^{6}$,J. Fluid Mech, vol 34, 625-39, 1968.

[2] Norberg, C., Effects of Reynolds number and a low-intensity freestream turbulence on the flow around a circular cylinder Publ 87/2, Dept. applied Thermo science \& fluid mechanics, Chalmers University of Technology, Sweden, 1987.

[3] Norberg, C., LDV-measurements in the near wake of a circular cylinder, Advances in Understanding of Bluff Body Wakes and Vortex-Induced Vibration, Washington D.C., 1998.

[4] Roshko, A., Experiments on the flow past a circular cylinder at very high Reynolds number, J. Fluid Mech., 10(3), 345-56, 1961.

[5] Catwell, B. \& Coles, D., An experimental study of entrainment and transport in the turbulent near wake of a circular cylinder, J. Fluid Mech, $136321-74,1983$.

[6] Lourenco, L. M. \& Shih, C., Characteristics of the plane turbulent near wake of a circular cylinder. A particle image velocimetry study. Data taken from [7].

[7] Ma. X, Karamanos, G. S. \& Karniadakis, G.E., Dynamics and lowdimensionality of a turbulent near wake, J. Fluid Mech, 410 29-65, 2000.

[8] Ong, L. \& Wallace, J., The velocity field of the turbulent very near wake of a circular cylinder, Exp. In Fluids, 20 441-53, 1996.

[9] Beaudan, P. \& Moin, P., Numerical experiments on the flow past a circular cylinder at a sub-critical Reynolds number, Report No. TF-62, Thermosciences Division, Dept. Mechanical Engineering, Stanford University, USA, 1994.

[10] Mittal, R. \& Moin, P., Suitability of upwind-biased finite difference schemes for large-eddy simulation of turbulent flow, AIAA J., 35 (8) 1415 $1417,1997$. 
[11] Breuer, M., Towards technical application of large eddy simulation, $Z$. Angew. Math. Mech, 81 (suppl 3), S461-S462, 2001.

[12] Liefvendahl, M., Bensow, R. \& Wikstrom, N., Propeller flow field analysis using Large Eddy Simulation, $26^{\text {th }}$ Symp. On Naval Hydrodynamics, Rome, Italy, 2006.

[13] Grinstein et al, Implicit Large Eddy Simulation, Computing Turbulent Fluid Dynamics, Cambridge University Press, 2007.

[14] Fureby, C., Large Eddy Simulation of Ship Hydrodynamics, 27 Symposium on Naval Hydrodynamics, Korea, 2009.

[15] Felice, D.F., Felli, M. \& Liefvendahl, M., Numerical and Experimental Analysis of the Wake Behavior of a Generic Submarine Propeller, First International Symposium on Marine Propulsors, 22-24 June, Trondheim, Norway, 2009.

[16] Wong, J. \& Png, E., Numerical Investigation of the Wing-Body Junction Vortex using various Large Eddy Simulation Models, $39^{\text {th }}$ AIAA Fluid Dynamics Conference, 22-25 June, San Antonio, Texas, 2009.

[17] Sagaut, P., Large Eddy Simulation for Incompressible Flows, Springer, 2000.

[18] Alin, N., Fureby, C., \& Svennberg, U., LES of the Flow past Simplified Submarine Hulls, $8^{\text {th }}$ International Conference on Numerical Ship Hydrodynamics, Sept 22-25, 2003, Busan, Korea.

[19] ANSYS-FLUENT v6.3, User's Guide.

[20] Fureby, C., Bensow, R. \& Persson, T., Scale Similarity Revisited in LES, Turbulent Shear Flow Phenomena IV, p1077, 2005.

[21] Wikstrom, N., Svennger, U., Alin, N. \& Fureby, C., Large eddy simulation of the flow around an inclined prolate spheroid, Journal of Turbulence, 2004.

[22] Franke, J. \& Frank, W., Large eddy simulation of the flow past a circular cylinder at $\mathrm{Re}_{\mathrm{D}}=3900, \mathrm{~J}$. Wind Engineering \& Industrial Aerodynamics, 90 p1191-1206, 2002.

[23] Persson, T., Bensow, R. \& Fureby, C., Flow around a Circular Cylinder at $\mathrm{Re}=3900$ and 140,000, $7^{\text {th }}$ Numerical Towing Tank Symposium, Hamburg, Germany, 2004. 